Original Research Paper

\title{
Application of Graph Theory Concepts in Computer Networks and its Suitability for the Resource Provisioning Issues in Cloud Computing-A Review
}

\author{
Kanniga Devi Rangaswamy and Murugaboopathi Gurusamy \\ Department of Computer Science and Engineering, Kalasalingam University, Krishnankoil, Tamilnadu, India
}

\author{
Article history \\ Received: 23-08-2017 \\ Revised: $30-11-2017$ \\ Accepted: 18-01-2018 \\ Corresponding Author: \\ Kanniga Devi Rangaswamy \\ Department of Computer \\ Science and Engineering, \\ Kalasalingam University, \\ Krishnankoil, Tamilnadu, India \\ Email: rkannigadevi@gmail.com
}

\begin{abstract}
Cloud computing, a kind of web service provisioning model, provides immense benefits over traditional IT service environments with the help of virtualization technology. As cloud computing is not a fully matured paradigm, it poses many open issues to be addressed. The key research problem in cloud computing is efficient resource provisioning which is due to its complex and distributed architecture. Graph-based representations of complex networks provide simpler views and graph theoretical techniques provide simpler solutions for lot of issues inherent in networks. Hence, this paper begins with exploration of graph theory applications in computer networks with specific focus on graph theory applications in cloud computing. This work pays attention to basic resource provisioning problems arise in cloud computing environments and presents some conceptual graph theoretical suggestions to address these issues.
\end{abstract}

Keywords: Computer Network, Cloud Computing, Graph Theory, Resource Provisioning

\section{Introduction}

The next step in the evolution of distributed computing is cloud computing. It inherits existing distributed computing models such as grid computing, utility computing and adds additional flavour namely virtualization. Large-scale processing and storage of data are very much simplified with the advent of costeffective cloud computing solutions. The Cloud Data Center (CDC) (Wu and Buyya, 2015) is very complex with resources distributed globally leading to several issues. The authors of (Zhang et al., 2010; Smith et al., 2009; Marston et al., 2011; Rimal et al., 2009; Shahzad, 2014) addressed several key and fundamental cloud computing issues like resource provisioning, security, privacy, energy and interoperability, but this list is not limited. From the perspective of cloud service provider and cloud service consumer, these issues provide different pictures. While cloud computing provides opportunities to migrate the IT business services online, these key issues need to be resolved before it is accepted as a successful business model.

This article identifies the opportunities of graph theory (Bondy and Murty, 1976) based solutions for the resource provisioning issues inherent in cloud computing. First, it starts with graph theory applications in various areas in computer networks and then explores its suitability to address the resource provisioning issues of cloud. Graph theory is part of discrete mathematics and useful structure to model relationship between objects. Graph theory mainly finds its applications in network modeling, biology, electrical network, computational algorithms and scheduling. Graph theoretical techniques are highly used by computer science applications especially in modeling and routing in networks. Representing a problem as a graph can provide a different point of view and makes a problem much simpler. It provides tools for solving the problem and set of techniques for analysing graphs.

This work has two main parts. The first part gives an overview of graph theory applications in computer networks. As cloud data center has a set of interconnected systems, graph theoretical solutions on computer networks can well be applied on cloud with suitable modifications to address its issues. The second part gives an overview of graph theory applications in cloud. The main idea behind this work is to find out the scope for applicability of graph theory to address resource provisioning issues in cloud. 


\section{Key Research Issues in Cloud Computing}

\section{Resource Provisioning}

The authors of (Meng et al., 2010; Buyya et al., 2011; Vecchiola et al., 2012; Javadi et al., 2012; Warneke and Kao, 2011) proposed that unlike traditional resource provisioning where resources are provisioned as it is, cloud demands efficient resource provisioning algorithms to provision virtualized resources to meet SLA requirements. Virtualized data centers are envisioned to provide better management flexibility, lower cost, scalability, better resources utilization and energy efficiency, but virtualization is not an easy task to do.

\section{Security}

The authors of (Denz and Taylor, 2013; Ward and Barker, 2014; Mahajan et al., 2011; Subashini and Kavitha, 2011; Zissis and Lekkas, 2012) discussed that as users and corporate information reside on third-party systems, no one can guarantee how secure the data are. It is prone to leakage of information and attack. Security is a primary issue which should be handled by all the cloud service providers to retain their business in the market. They should take steps to protect data and its privacy. Five most representative security and privacy attributes are confidentiality, integrity, availability, accountability and privacy-preservability.

\section{Cost}

The authors of (Sahni and Vidyarthi, 2015; Tsai and Liao, 2015; Waibel et al., 2016; Hadji et al., 2016) proposed that the public cloud offers pay per use, which can provide low-cost options for short-term projects. Still, for long-term use, enterprise IT organizations may be better off making a capital investment to purchase additional hardware and software. Enterprises need to conduct a break-even analysis to determine whether a public or private cloud would be more cost-effective for them. From the perspective of provider, they are interested in customer satisfaction and generating revenue out of their services. From the perspective of consumer, they are interested in cost-effective solutions. To balance these two points, cost-effective cloud solutions need to be developed.

\section{Reliability}

The authors of (Vishwanath and Nagappan, 2010; Poola et al., 2014; Di and Wang, 2013; Zheng et al., 2012) stated that due to high system complexity and distributed structure, even carefully engineered data centers are subject to a large number of failures. Fault tolerant systems should be built to address reliability concerns. Because of the abstraction nature of cloud environment, there arise a need to develop new or extend traditional fault tolerant approaches. VM migration and
Server consolidation are the major threatening factor for fault tolerance as they incur service downtime.

\section{Interoperability}

The authors of (Govindrajan and Lakshmanan, 2010; Loutas et al., 2011) stated that interoperability of heterogeneous cloud platforms are difficult because they use distinct hypervisor and VM technologies. The platforms also use various security standards and management interfaces. Multiple vendors with different product standards poses challenges for interoperability. Cloud adoption will be stopped if there is not a good way of integrating data and applications across clouds; hence a unified cloud interface and open standards need to be developed.

\section{Energy}

The authors of (Beloglazov et al., 2011; Guo and Fang, 2013; Dayarathna et al., 2016; Beloglazov and Buyya, 2010; Lee and Zomaya, 2010) stated that increasing demand for computational power, leads to setting up large-scale data centers. On the other side, the power consumption of these large-scale data centers is enormous. Hence, design of energy efficient hardware and intelligent resource management techniques is required. Due to enormous power consumption, carbon dioxide $\left(\mathrm{CO}_{2}\right)$ emission is also more contributing to the greenhouse effect. Hence number of practices need to be applied to achieve energy efficiency, such as improvement of applications' algorithms, energy efficient hardware and energy-efficient resource management strategies on a virtualized data center.

Out of all these above mentioned key issues, this work pays much attention to resource provisioning issue and application of graph theory on it.

\section{Resource Provisioning in Cloud}

In cloud computing, resource provisioning (Meng et al., 2010; Buyya et al., 2011; Vecchiola et al., 2012; Javadi et al., 2012; Warneke and Kao, 2011) is the allocation of a cloud data center resources to a user. When cloud data center accepts requests for hardware or software resources, it must create and provision them as virtualized resources. It means the monitoring, dynamic selection/scheduling, deployment/placement and management/load balancing of software and hardware resources for ensuring Service Level Agreement (SLA). The SLA is an agreement between the cloud service provider and the user on guaranteeing Quality of Service (QoS). The provisioning can be done in several different ways. In particular, this work addresses the following aspects of resource provisioning from $\mathrm{CDC}$ :

- $\quad$ Efficient monitoring for provisioning CDC resources

- Optimal VM placement and migration in CDC for energy-efficient resource provisioning 
- Proper locating of CDCs and allocation of CDCs to the source of requests

- Clustering distributed CDCs for faster server provisioning

- Uniform assignment of clients to CDC servers

- Traffic-aware VM migration to load balance cloud servers

\section{Efficient Monitoring for Provisioning CDC Resources}

Task scheduling, load balancing are complicated in cloud computing environment due to its abstract heterogeneous architecture, dynamic behaviour and resource heterogeneity. Monitoring of resources is required before performing scheduling and load balancing.

Optimal VM Placement and Migration in CDC for Energy-Efficient Resource Provisioning

Keeping lot of PMs and VMs running in the datacenter consumes more energy, leading to higher operating costs. Hence identifying physical machines with least load and migrating its load to some other physical machines and then shutting them down saves energy. Conservation of energy may be better achieved through optimal placement of VMs on the PMs and performing VM migrations, so that energy consumption may be maintained at desirable level.

Proper Locating of CDCs and Allocation of CDCs to the Source of Requests

The requests for the CDC services can come from different parts of the world. The term source of requests/clients denote the users who make requests to various cloud data center services. The distance between the cloud data center and the source of requests is a major factor influencing the quality of service in terms of response time and latency. Cloud data center allocation is one of the major issues in cloud computing. An efficient allocation of cloud data center to the source of requests may improve the quality of services.

\section{Clustering Distributed CDCs for Faster Server Provisioning}

Normally cloud data centers are distributed across the world to increase the availability of services by remote mirroring, replication which are the kind of redundancy mechanisms. It is distributed mainly for disaster recovery. Clustering region-wise deployed cloud data centers will provide rapid responses.

\section{Uniform assignment of clients to CDC servers}

In a distributed cloud data center environment, load balancing techniques direct the requests to the closest source or to the source with the most able capacity to serve the request. Variety of algorithms are used to perform load balancing. But there is a trade-off always exits between choosing the closest cloud data center and balancing the load of cloud data center. Sometimes a cloud data center closer to the user location may be in overloaded condition, during this case, the requests will be routed to a distant cloud data center which is capable of handling the requests. Hence, there arise a need to deal with this trade-off which may consider both proximity and load at the same time.

\section{Traffic-Aware VM Migration to Load Balance Cloud Servers}

Upon receiving the load information, cloud broker must invoke load balancing procedure to distribute the load uniform across the hosts in the CDC. It can be done by migrating some of the VMs from overloaded hosts to underloaded hosts considering only server-side constraints. Network-side constraints also need to be considered to enhance the performance of CDCs.

\section{Graph Theory Applications in Computer Networks}

This section summary of some of the works, which applied graph theory in various types of computer network.

Table 1 lists some of the possible graph theory applications in various types of network. Since cloud is a kind of network, these traditional graph theoretical techniques can be analyzed for their suitability to address resource provisioning issues in cloud and also presents some conceptual suggestions for it.

\section{Graph Theory Applications in Cloud Computing}

This section provides summary of works, which applied graph theory in cloud.

The author of (Çatalyürek et al., 2011) proposed a heuristic based on hypergraph and its partitioning for optimizing scientific workflow execution. The author of (Dai et al., 2009) modeled the reliability of the cloud services. This paper models network failure, overflow failure, timeout failure, resource missing failure, hardware failure, software failure and database failure failures using Markov models, Queuing Theory and Graph Theory. The author of (Verbelen et al., 2013) investigated how to optimally deploy software applications on the offered infrastructure in the cloud, by minimizing the network usage. Especially in the context of mobile computing. They designed and evaluated graph partitioning algorithms that allocate software components to machines in the cloud while minimizing the required bandwidth. The author of (Li et al., 2012) proposed CAM, a cloud platform that provides an innovative resource scheduler particularly designed for hosting MapReduce applications in the cloud. 
Table 1: Graph theory in computer networks

\begin{tabular}{|c|c|c|c|}
\hline Author & Graph theory technique & Issue addressed & Type of network \\
\hline Wang et al. (2006; & \multirow[t]{3}{*}{ Graph coloring } & Interference reduction, Interference Aware & \multirow{9}{*}{$\begin{array}{l}\text { Wireless networks-Mobile } \\
\text { Adhoc Networks and Sensor } \\
\text { networks }\end{array}$} \\
\hline Tamura and Nakano, 2011; & & TDMA Link scheduling, Job Scheduling/ & \\
\hline Tamura et al., 2008) & & Assignment problems, Resource allocation & \\
\hline Alzoubi et al. (2002; & Dominating set and & Routing, fault tolerance, energy- efficiency, & \\
\hline Scheideler et al., 2008; & Connected dominating set & delivery delay reduction, connectedness, & \\
\hline Wu and Li, 1999; Erciyes et al., & & Virtual backbone construction for efficient & \\
\hline 2007; Liu et al., 2007; & & routing, overlay network construction, & \\
\hline Dai and Wu 2004; & & Search space reduction, Clustering nodes & \\
\hline Muhammad 2007) & & & \\
\hline \multirow{2}{*}{ Kawahigashi et al. (2005) } & Random graphs & Connectivity, scalability, Routing, & \\
\hline & & Congestion handling, Modeling the network & \\
\hline Meghanathan (2012) & Shortest path algorithms & Route computation for communication & \\
\hline Wang and Li (2006) & Spanner & Minimum power assignment & \\
\hline Dong et al. (2010) & Topological graph & Network Coverage problem & \\
\hline Newsome and Song (2003) & Graph embedding & Routing & \\
\hline Prasanna et al. (2014) & Graph labeling & Fast communication via radio labeling & \\
\hline Bose et al. (2001) & Gabriel graph, unit graph & Routing with guaranteed delivery & \\
\hline Xie et al. (2010) & DAG & Workflow modeling, Loop-free routing & \\
\hline Xie et al. (2014) & DAG & Task Scheudling & Network embedded systems \\
\hline Nakayama and Koide (2013) & Network flow & Flow optimization & Mesh network \\
\hline Fatmi and Pan (2014) & Tree & Data center/ network modeling & Datacenter \\
\hline Bunke et al. (2006) & Graph matching & $\begin{array}{l}\text { Abnormal event detection in network } \\
\text { by graph comparison }\end{array}$ & General network \\
\hline Jana and Naik (2009) & $\begin{array}{l}\text { Spanning and minimum } \\
\text { spanning tree algorithms }\end{array}$ & Loop-free connectedness, clustering & \\
\hline Wang et al. (2012) & Graph traversal & Searching an object & $\begin{array}{l}\text { General network query } \\
\text { optimization }\end{array}$ \\
\hline $\begin{array}{l}\text { Maldeniya et al. }(2013 ; \\
\text { Tang et al., 2008) }\end{array}$ & Graph partitioning & Clustering, faster communication & $\begin{array}{l}\text { Real road networks, } \\
\text { World Wide Web }\end{array}$ \\
\hline Dai et al. $(2007 ; 2009)$ & Virtualized graph model & Grid service reliability evaluation & Grid \\
\hline Venugopal and Buyya (2006) & Set covering problem & Mapping applications with data sets & \\
\hline Xiao and Parhami (2003) & Cayley digraphs & Design of scalable Interconnection networks & \\
\hline Bentley (1979) & KD-Tree & Multi-dimensional search & Database \\
\hline Potluri and Singh (2012) & Capacitated dominating set & $\begin{array}{l}\text { Minimum capacitated dominating } \\
\text { set construction }\end{array}$ & Wireless networks \\
\hline Bar-Ilan et al. (1992) & Facility location problem & Locating centers & General facility \\
\hline
\end{tabular}

Table 2: Graph theory in cloud

\begin{tabular}{|c|c|c|c|}
\hline Author & Graph theory technique & Issue addressed & Limitation \\
\hline $\begin{array}{l}\text { Çatalyürek et al. } \\
\text { (2011) }\end{array}$ & Hypergraph & Modeling cloud network & $\begin{array}{l}\text { Scheduling is done in two-phases which increases makespan. If } \\
\text { done in single phase, it may minimize the makespan of the } \\
\text { workflow. This work didn't consider dynamic workflows whose } \\
\text { execution pattern changes over time as well. }\end{array}$ \\
\hline Dai et al. (2009) & Spanning tree & Cloud service reliability & $\begin{array}{l}\text { Cloud service reliability model and evaluation algorithm have } \\
\text { not been validated by simulation and real-life data. They have } \\
\text { proposed only theoretical model. }\end{array}$ \\
\hline $\begin{array}{l}\text { Verbelen et al. } \\
\text { (2013) }\end{array}$ & Graph partitioning & $\begin{array}{l}\text { Software deployment in } \\
\text { mobile cloud }\end{array}$ & $\begin{array}{l}\text { This work considered only minimizing the bandwidth between } \\
\text { software components. It didn't minimize the execution time } \\
\text { of tasks. This work provide scope for integrating their algorithms } \\
\text { to address energy consumption objective. }\end{array}$ \\
\hline Li et al. (2012) & $\begin{array}{l}\text { Flow network algorithms } \\
\text {-Ford-Fulkerson }\end{array}$ & $\begin{array}{l}\text { Optimizing MapReduce } \\
\text { performance in cloud }\end{array}$ & $\begin{array}{l}\text { Validation of min-cost flow model for data and VM placement } \\
\text { is to be done in larger real VM clusters. }\end{array}$ \\
\hline Binz et al. (2012) & Topological graph theory & $\begin{array}{l}\text { Topology management } \\
\text { in cloud }\end{array}$ & $\begin{array}{l}\text { Enterprise Topology Graph (ETG) is to be built as reusable } \\
\text { building blocks to address specific problem domains with } \\
\text { broad set of basic operations. }\end{array}$ \\
\hline Bansal et al. (2011) & Graph mapping & $\begin{array}{l}\text { Congestion minimization } \\
\text { in cloud network }\end{array}$ & $\begin{array}{l}\text { Only mapping of two classes of workloads, namely depth-d } \\
\text { trees and complete graph are considered. }\end{array}$ \\
\hline Chan et al. (2009) & Predicate-based graph & $\begin{array}{l}\text { Modeling and Testing } \\
\text { cloud }\end{array}$ & $\begin{array}{l}\text { This model supports only stateless atomic operations expressed } \\
\text { in context free grammar. Some other attributes such as scalability, } \\
\text { exception handling, dynamic binding, service interactions can also } \\
\text { be considered to make the model valuable. }\end{array}$ \\
\hline Peng et al. (2015) & Graph cut theory & $\begin{array}{l}\text { Virtual Machine (VM) } \\
\text { clustering in cloud }\end{array}$ & $\begin{array}{l}\text { Time taken for VM clustering is a valuable parameter, which } \\
\text { is not mentioned. Not mentioned the applicability of }\end{array}$ \\
\hline $\begin{array}{l}\text { Zegzhda and } \\
\text { Nikolsky (2014) }\end{array}$ & Graph model & $\begin{array}{l}\text { Security model for VM } \\
\text { hypervisor in cloud }\end{array}$ & $\begin{array}{l}\text { hypervisor security model on the specific type of hypervisor } \\
\text { architectures, namely hosted or bare-metal. }\end{array}$ \\
\hline
\end{tabular}


CAM uses a flow-network-based algorithm that is able to optimize MapReduce performance under the specified constraints. The author of (Binz et al., 2012) proposed Enterprise Topology Graphs (ETG) as formal model to describe an enterprise topology. The author of (Bansal et al., 2011) represented the physical network of the cloud as a graph considering the objective of minimizing the congestion. The author of (Chan et al., 2009) formulated a computing cloud as a kind of graph, a computing resource such as services or intellectual property access rights as an attribute of a graph node and the use of the resource as a predicate on an edge of the graph. It also proposes to model cloud computation as a set of paths in a subgraph of the cloud such that every edge contains a predicate that is evaluated to be true. Finally, it presents a set of algorithms to compose cloud computations and model-based testing criteria to test cloud applications. The author of (Peng et al., 2015) constructed a method for VM cluster by energy minimization based on graph theory. Then, they changed deployment of VM cluster into maximum flow minimum cut problem and finally cut formed for VM cluster. The author of (Zegzhda and Nikolsky, 2014) described a formal security model for virtual machine hypervisors in cloud systems based on the graph theory.

From Table 2, it is observed that only a very minimal literature is available on graph theory applications in cloud and most of these works overlooked its application on resource provisioning. Thus, it opens lot of opportunities to apply graph theoretical techniques to address resource provisioning issues in cloud.

The following section presents some conceptual suggestions for applying graph theory for resource provisioning issues in cloud computing.

\section{Some Conceptual Suggestions for the Suitability of Graph Theory Techniques for Addressing Resource Provisioning in Cloud}

This section considers some of the graph theoretical techniques from Table 1 and suggests the following aspects of resource provisioning in cloud and the suitability of such graph theoretical models and techniques to address its issues.

\section{How to Perform Efficient Monitoring for Provisioning CDC Resources?}

Monitoring the complex, distributed cloud architecture for tracking resource status is a challenging task. Monitoring ensures the availability of resources before performing successful provisioning. Generally, a central monitor keeps track of the resources in cloud (Galstad, 2016).

The monitor has to query all the resources in the network periodically to get their availability status, which increases network/message overhead. To resolve this, this work suggests to represent cloud as a graph and construct a minimum dominating set structure, which can be used for monitoring resource status. The minimum dominating set structure can minimize the number of message updates made to the monitor and minimize the update time compared to traditional monitoring methods.

How to Perform Optimal VM Placement and Migration in CDC for Energy-Efficient Resource Provisioning?

As VM placement has direct impact on the performance of the $\mathrm{CDC}$, this work suggests to optimize VM placement and VM migration for energy-efficient resource provisioning. For optimal VM placement, it suggests to construct KD-tree structure of cloud servers and VMs will be placed on best-fit cloud servers quickly. This KD-tree is a useful structure when search involves a multidimensional search key and it is known as associative search. KD-tree is defined as a tree for storing the values or objects in multidimensional space. They scale well in high dimensions. All the resource dimensions namely CPU, RAM, storage and bandwidth of cloud servers and VM can well be represented using KD-tree structure. Moreover, this structure helps in identifying least loaded PMs easily so that VMs running on it can be migrated to other cloud servers and powered off to conserve energy. The author of (Mandal and Khilar, 2013) constructed one dimensional Binary Search Tree, which considers only single resource dimension, $\mathrm{CPU}$.

How to Ensure Proper Locating of CDCs and Allocation of CDCs to the Source of Requests?

This suggests to model cloud data center network as a graph and use facility location problem to locate CDCs in appropriate locations to serve the source of requests. Limiting the distance between cloud data centers and the source of requests leads to faster service provisioning. The author of (Doyle et al., 2013) has proposed the source of requests assignment to the closest cloud data center to reduce the carbon emission but they modeled cloud data center as a complete graph which is unrealistic. They modelled both the networking and computational components of the infrastructure as a graph and proposed a system which utilizes Voronoi partitions to determine how source requests to be routed to appropriate data center based on the relative priorities of the cloud operator for latency purposes. The author of (Bar-Ilan et al., 1992) provided solutions for facility location problems. They have considered distributing the clients to centers as balanced as possible, but they have overlooked the distance between clients and centers, which is also essential for faster service provisioning. 
How to Cluster Distributed CDCs for Faster Server Provisioning?

This suggests to model the cloud environment as a graph and cluster distributed CDCs in order to enable faster service provisioning to the clients. Many works have considered graph-based K-Means (Galluccio et al., 2012) and K-Spanning tree (Zhou et al., 2011) clustering methods to cluster a network. In all these approaches the number of clusters $\mathrm{K}$ to be created should be known in advance, but determining $\mathrm{K}$ is impractical in case of distributed CDCs. Hence, this work suggests to use dominating set based clustering, where each dominating node acts as a cluster head and neighbors are connected to these cluster heads which makes service provisioning fast. The number of clusters $(\mathrm{K})$ to be created is determined based on size of the graph. So, it leads to a non-constrained clustering algorithm.

How to Ensure Uniform Assignment of Clients to CDC Servers?

This work suggests to use capacitated dominating set concept (Potluri and Singh, 2012) to assign clients to CDC servers. The capacitated dominating set is the problem of finding a dominating set with the additional constraint that the nodes dominated do not exceed the capacity of the dominating node. The capacity can be uniform across all nodes or variable. Hence, a uniform capacity can be assigned to a homogeneous CDC environment and a variable capacity can be assigned to a heterogeneous CDC environment. For client assignment to CDCs, exiting methods have used Round Robin (Radojević and Žagar, 2011) and its variant approaches, which may not guarantee uniformity all the times.

\section{How to Efficiently Perform Traffic-Aware VM} Migration to Load Balance Cloud Servers?

Network flow problem (Nakayama and Koide, 2013) in the form of Maximum flow and Minimum cut problem finds its application in various fields ranging from checking network connectivity, network reliability, namely a few.

This work suggests to use network flow problem by converting cloud into a flow network and to perform traffic-aware live VM migration in order to load balance the cloud servers. Most of the existing benchmarked VM migration approaches (Khanna et al., 2006; Wood et al., 2007) consider only host-side resource constraints with little consideration on network-side constraints. A good VM migration algorithm which considers both serverside and network-side constraints can greatly improve their performance. This can be done on both live migration or cold migration scenarios.

In network flow problem, a set of path in a graph $\mathrm{G}$ is edge-disjoint if each edge in $G$ appears in at most one path. In cloud, some $\mathrm{k}$ number of edge-disjoint paths can be computed between overloaded and underloaded servers and can migrate each VM from overloaded server to underloaded server on one of these $\mathrm{k}$ paths to perform fast and traffic-aware VM migrations.

\section{Conclusion and Future Work}

In this study, an analysis is done on the application of graph theory concepts in computer networks and its suitability to address resource provisioning issues in cloud. In future, we would like to analyze its scope in other research areas of cloud computing.

\section{Acknowledgment}

The authors would like to thank the support rendered by Kalasalingam University to carry out this review work.

\section{Author's Contributions}

Kanniga Devi Rangaswamy: She devised the main conceptual ideas and developed the review framework. She contributed to part of the literature collection and drafted the manuscript in consultation with Murugaboopathi Gurusamy.

Murugaboopathi Gurusamy: He supervised this review work. He contributed to part of the literature collection and to the formalism of review work. He provided critical feedback and helped shape the research and manuscript.

\section{Ethics}

This research manuscript is original and the manuscript is not published elsewhere. The corresponding author ensure that all authors have read and approved the manuscript and no ethical issues involved.

\section{References}

Alzoubi, K.M., P.J. Wan and O. Frieder, 2002. Messageoptimal connected dominating sets in mobile ad hoc networks. Proceedings of the 3rd ACM International Symposium on Mobile Ad Hoc Networking and Computing, Jun. 09-11, ACM, Lausanne, Switzerland, pp: 157-164.

DOI: $10.1145 / 513800.513820$

Bansal, N., K.W. Lee, V. Nagarajan and M. Zafer, 2011. Minimum congestion mapping in a cloud. Proceedings of the 30th Annual ACM SIGACTSIGOPS Symposium on Principles of Distributed Computing, Jun. 06-08, ACM, San Jose, California, USA, pp: 267-276. DOI: 10.1145/1993806.1993854

Bar-Ilan, J., G. Kortzars and D. Pelag, 1992. How to allocate network centers. J. Algorithms, 15: 385-415. DOI: $10.1006 /$ jagm.1993.1047 
Beloglazov, A. and R. Buyya, 2010. Energy efficient resource management in virtualized cloud data centers. Proceedings of the 10th IEEE/ACM International Conference on Cluster, Cloud and Grid Computing, May 17-20, IEEE Xplore Press, Melbourne, VIC, Australia, pp: 826-831. DOI: 10.1109/CCGRID.2010.46

Beloglazov, A., R. Buyya, Y.C. Lee and A. Zomaya, 2011. A taxonomy and survey of energy-efficient data centers and cloud computing systems. Adv. Comput., 82: 47-111.

DOI: 10.1016/B978-0-12-385512-1.00003-7

Bentley, J.L., 1979. Multidimensional binary search trees in database applications. IEEE Trans. Software Eng., 5: 333-340. DOI: 10.1109/TSE.1979.234200

Binz, T., C. Fehling, F. Leymann, A. Nowak and D. Schumm, 2012. Formalizing the cloud through enterprise topology graphs. Proceedings of the IEEE 5th International Conference on Cloud Computing, Jun. 24-29, IEEE Xplore Press, Honolulu, HI, USA, pp: 742-749. DOI: 10.1109/CLOUD.2012.143

Bondy, J.A. and U.S.R. Murty, 1976. Graph Theory with Applications. 1st Edn., North Holland, New York, ISBN: 0444194517, pp: 264.

Bose, P., P. Morin, I. Stojmenović and J. Urrutia, 2001. Routing with guaranteed delivery in ad hoc wireless networks. Wireless Netw., 7: 609-616.

DOI: $10.1006 /$ jagm.1993.1047

Bunke, H., P. Dickinson, A. Humm, C. Irniger and M. Kraetzl, 2006. Computer network monitoring and abnormal event detection using graph matching and multidimensional scaling. Proceedings of the 6th Industrial Conference on Data Mining Conference on Advances in Data Mining: Applications in Medicine, Web Mining, Marketing, Image and Signal Mining, Jul. 14-15, Springer, Leipzig, Germany, pp: 576-590. DOI: 10.1007/11790853 45

Buyya, R., S.K. Garg and R.N. Calheiros, 2011. SLAoriented resource provisioning for cloud computing: Challenges, architecture and solutions. Proceedings of the International Conference on Cloud and Service Computing, Dec. 12-14, IEEE Xplore Press, Hong Kong, China, pp: 1-10.

DOI: 10.1109/CSC.2011.6138522

Çatalyürek, U.V., K. Kaya and B. Uçar, 2011. Integrated data placement and task assignment for scientific workflows in clouds. Proceedings of the 4th International Workshop on Data-Intensive Distributed Computing, Jun. 08-08, ACM, San Jose, California, USA, pp: 45-54. DOI: 10.1145/1996014.1996022

Chan, W.K., L. Mei and Z. Zhang, 2009. Modeling and testing of cloud applications. Proceedings of the IEEE Asia-Pacific Services Computing Conference, Dec. 7-11, IEEE Xplore Press, Singapore, pp: 111-118. DOI: 10.1109/APSCC.2009.5394131
Dai, F. and J. Wu, 2004. An extended localized algorithm for connected dominating set formation in ad hoc wireless networks. IEEE Trans. Parallel Distributed Syst., 15: 908-920. DOI: 10.1109/TPDS.2004.48

Dai, Y.S., B. Yang, J. Dongarra and G. Zhang, 2009. Cloud service reliability: Modeling and analysis. Innovative Computing Laboratory, Department of Electrical Engineering and Computer Science, University of Tennessee, Knoxville.

Dai, Y.S., Y. Pan and X. Zou, 2007. A hierarchical modeling and analysis for grid service reliability. IEEE Trans. Comput., 56: 681-691.

DOI: $10.1109 /$ TC.2007.1034

Dayarathna, M., Y. Wen and R. Fan, 2016. Data center energy consumption modeling: A survey. IEEE Commun. Surveys Tutorials, 18: 732-794. DOI: 10.1109/COMST.2015.2481183

Denz, R. and S. Taylor, 2013. A survey on securing the virtual cloud. J. Cloud Comput.: Adv. Syst. Applic., 2: 17-17. DOI: 10.1186/2192-113X-2-17

Di, S. and C.L. Wang, 2013. Error-tolerant resource allocation and payment minimization for cloud system. IEEE Trans. Parallel Distributed Syst., 24: 1097-1106. DOI: 10.1109/TPDS.2012.309

Dong, D., Y. Liu, K. Liu and X. Liao, 2010. Distributed coverage in wireless ad hoc and sensor networks by topological graph approaches. Proceedings of the IEEE 30th International Conference on Distributed Computing Systems, Jun. 21-25, IEEE Xplore Press, Genova, Italy, pp: 1417-1428. DOI: 10.1109/ICDCS.2010.9

Doyle, J., R. Shorten and D. O'Mahonym, 2013. Stratus: Load balancing the cloud for carbon emissions control. IEEE Trans. Cloud Comput., 1: 1-1. DOI: $10.1109 /$ TCC. 2013.4

Erciyes, K., O. Dagdeviren, D. Cokuslu and D. Ozsoyeller, 2007. Graph theoretic clustering algorithms in mobile ad hoc networks and wireless sensor networks survey. Applied Comput. Math., 6: 162-180.

Fatmi, O. and D. Pan, 2014. Distributed multipath routing for data center networks based on stochastic traffic modeling. Proceedings of the IEEE 11th International Conference on Networking, Sensing and Control, Apr. 7-9, IEEE Xplore Press, Miami, FL, USA, pp: 536-541. DOI: 10.1109/ICNSC.2014.6819683

Galluccio, L., O. Michel, P. Comon and A.O. Hero, 2012. Graph based K-means clustering. Signal Process., 92: 1970-1984. DOI: 10.1016/j.sigpro.2011.12.009

Galstad, E., 2016. Nagios NRPE Documentation.

Govindrajan, A. and Lakshmanan, 2010. Overview of Cloud Standards Cloud Computing. 1st Edn., Springer London.

Guo, Y. and Y. Fang, 2013. Electricity cost saving strategy in data centers by using energy storage. IEEE Trans. Parallel Distributed Syst., 24: 1149-1160. DOI: 10.1109/TPDS.2012.201 
Hadji, M., B. Aupeti and D. Zeghlache, 2016. Costefficient algorithms for critical resource allocation in cloud federations. Proceedings of the 5 th IEEE International Conference on Cloud Networking, Oct. 3-5, IEEE Xplore Press, pp: 1-6. DOI: 10.1109/CloudNet.2016.11

Jana, P.K. and A. Naik, 2009. An efficient minimum spanning tree based clustering algorithm. Proceedings of the International Conference on Methods and Models in Computer Science, Dec. 14-15, IEEE Xplore Press, Delhi, India, pp: 1-5. DOI: 10.1109/ICM2CS.2009.5397966

Javadi, B., J. Abawajy and R. Buyya, 2012. Failureaware resource provisioning for hybrid Cloud infrastructure. J. Parallel Distributed Comput., 72: 1318-1331. DOI: 10.1016/j.jpdc.2012.06.012

Kawahigashi, H., Y. Terashima, N. Miyauchi and T. Nakakawaji, 2005. Modeling ad hoc sensor networks using random graph theory. Proceedings of the 2nd IEEE Consumer Communications and Networking Conference, Jan. 6-6, IEEE Xplore Press, Las Vegas, NV, USA, pp: 104-109.

DOI: $10.1109 /$ CCNC.2005.1405152

Khanna, G., K. Beaty, G. Dhar and A. Kochu, 2006. Application performance management in virtualized server environments. Proceedings of the 10th IEEE/IFIP Network Operations and Management Symposium, Apr. 3-7, IEEE Xplore Press, Vancouver, BC, Canada, pp: 373-381. DOI: 10.1109/NOMS.2006.1687567

Lee, Y.C. and A.Y. Zomaya, 2010. Energy efficient utilization of resources in cloud computing systems. J. Supercomput., 60: 268-280. DOI: $10.1007 / \mathrm{s} 11227-010-0421-3$

Li, M., D. Subhraveti, A.R. Butt, A. Khasymski and P. Sarkar, 2012. CAM: A topology aware minimum cost flow based resource manager for mapreduce applications in the cloud. Proceedings of the $21 \mathrm{st}$ International Symposium on High-Performance Parallel and Distributed Computing, Jun. 18-22, ACM, Delft, The Netherlands, pp: 211-222. DOI: $10.1145 / 2287076.2287110$

Liu, D., X. Jia and I. Stojmenovic, 2007. Quorum and connected dominating sets based location service in wireless ad hoc, sensor and actuator networks. Comput. Commun., 30: 3627-3643. DOI: 10.1016/j.comcom.2007.07.001

Loutas, N., E. kamateri and E. Tarabanis, 2011. A semantic interoperability framework for cloud platform as a service. Proceedings of the IEEE 3rd International Conference on Cloud Computing Technology and Science, Nov. 29-Dec. 1, IEEE Xplore Press, Athens, Greece, pp: 280-287.

DOI: 10.1109/CloudCom.2011.45
Mahajan, P., S. Setty, S. Lee, A. Clement and L. Alvisi et al., 2011. Depot: Cloud storage with minimal trust. ACM Trans. Comput. Syst. DOI: $10.1145 / 2063509.2063512$

Maldeniya, S.L., A.S. Atukorale and W.W. Vithanage, 2013. Network data classification using graph partition. 19th Proceedings of the IEEE International Conference on Networks, Dec. 11-13, IEEE Xplore Press, Singapore, pp: 1-6. DOI: $10.1109 /$ ICON.2013.6781952

Mandal, S.K. and P.M. Khilar, 2013. Efficient virtual machine placement for on-demand access to infrastructure resources in cloud computing. Int. J. Comput. Applic., 68: 6-11.

Marston, S., Z. Li, S. Bandyopadhyay, J. Zhang and A. Ghalsasi, 2011. Cloud computing-the business perspective. Decision Support Syst., 51: 176-189. DOI: $10.1016 /$ j.dss.2010.12.006

Meghanathan, N., 2012. Graph theory algorithms for mobile ad hoc networks. Informatica, 36: 185-200. DOI: 10.4018/978-1-4666-0080-5.ch004

Meng, X., C. Isci, J. Kephart, L. Zhang and E. Bouillet et al., 2010. Efficient resource provisioning in compute clouds via VM multiplexing. Proceedings of the 7th International Conference on Autonomic Computing, Jun. 07-11, ACM, Washington, DC, USA, pp: 11-20. DOI: $10.1145 / 1809049.1809052$

Muhammad, R.B., 2007. A distributed graph algorithm for geometric routing in ad hoc wireless networks. J. Netw., 2: 50-57.

Nakayama, K. and T. Koide, 2013. A decentralized algorithm for network flow optimization in mesh networks. Proceedings of the IEEE Global Communications Conference, Dec. 9-13, IEEE Xplore Press, Atlanta, GA, USA, pp: 1532-1537. DOI: 10.1109/GLOCOM.2013.6831291

Newsome, J. and D. Song, 2003. GEM: Graph EMbedding for routing and data-centric storage in sensor networks without geographic information. Proceedings of the 1st International Conference on Embedded Networked Sensor Systems, Nov. 05-07, ACM, Los Angeles, California, USA, pp: 76-88. DOI: $10.1145 / 958491.958501$

Peng, Z., B. Xu, D. Cui, W. Lin and X.A. Wang, 2015. Deployment method of virtual machine cluster based on energy minimization and graph cuts theory. Proceedings of the 10th International Conference on P2P, Parallel, Grid, Cloud and Internet Computing, Nov. 4-6, IEEE Xplore Press, Krakow, Poland, pp: 800-803. DOI: 10.1109/3PGCIC.2015.51

Poola, D., K. Ramamohanarao and R. Buyya, 2014. Fault-tolerant workflow scheduling using spot instances on clouds. Proc. Comput. Sci., 29: 523-533. DOI: 10.1016/j.procs.2014.05.047 
Potluri, A. and A. Singh, 2012. A greedy heuristic and its variants for minimum capacitated dominating set. Proceedings of the International Conference on Contemporary Computing, (CCC' 12), Springer, Berlin, Heidelberg, pp: 28-39.

DOI: 10.1007/978-3-642-32129-0_9

Prasanna, N.L., K. Sravanthi and N. Sudhakar, 2014. Applications of graph labeling in major areas of computer science. Int. J. Res. Comput. Commun. Technol., 3: 819-823.

Radojević, B. and M. Žagar, 2011. Analysis of issues with load balancing algorithms in hosted (cloud) environments. Proceedings of the 34th International Convention MIPRO, May 23-27, IEEE Xplore Press, Opatija, Croatia, pp: 416-420.

Rimal, B.P., E. Choi and I. Lumb, 2009. A taxonomy and survey of cloud computing systems. Proceedings of the 5th International Joint conference on INC, IMS and IDC, IEEE Xplore Press, Seoul, South Korea, pp: 44-51.

DOI: 10.1109/NCM.2009.218

Sahni, J. and D.P. Vidyarthi, 2015. A cost-effective deadline-constrained dynamic scheduling algorithm for scientific workflows in a cloud environment. IEEE Trans. Cloud Comput.

DOI: 10.1109/TCC.2015.2451649

Scheideler, C., A.W. Richa and P. Santi, 2008. An O(log n) dominating set protocol for wireless ad-hoc networks under the physical interference model. Proceedings of the 9th ACM International Symposium on Mobile Ad Hoc Networking and Computing, May 26-30, ACM, Hong Kong, Hong Kong, China, pp: 91-100.

DOI: $10.1145 / 1374618.1374632$

Shahzad, F., 2014. State-of-the-art survey on cloud computing security challenges, approaches and solutions. Proc. Comput. Sci., 37: 357-362. DOI: $10.1016 /$ j.procs.2014.08.053

Smith, D.M., D.W. Cearley and D.C. Plummer, 2009. Key issues for cloud computing. Gartner.

Subashini, S. and V. Kavitha, 2011. A survey on security issues in service delivery models of cloud computing. J. Netw. Comput. Applic., 34: 1-11. DOI: $10.1016 /$ j.jnca.2010.07.006

Tamura, H. and K. Nakano, 2011. On applications of graph/network theory to problems in communication systems. ECTI Trans. Comput. Inform. Technol., 5: $15-21$.

Tamura, H., M. Sengoku and S. Shinoda, 2008. Edge coloring problem of graph theory considering interference on network coding. Proceedings of the 23rd International Technical conference on Circuits/Systems, Computers and Commnications, (SCC' 08), pp: 301-304.
Tang, Y., Y. Zhang and H. Chen, 2008. A parallel shortest path algorithm based on graph-partitioning and iterative correcting. Proceedings of the 10th IEEE International Conference on High Performance Computing and Communications, Sept. 25-27, IEEE Xplorev Press, Dalian, China, pp: 155-161.

DOI: 10.1109/HPCC.2008.113

Tsai, L. and W. Liao, 2015. StarCube: An on-demand and cost-effective framework for cloud data center networks with performance guarantee. IEEE Trans. Cloud Comput. DOI: $10.1109 /$ TCC.2015.2464818

Vecchiola, C., R.N. Calheiros, D. Karunamoorthy and R. Buyya, 2012. Deadline-driven provisioning of resources for scientific applications in hybrid clouds with Aneka. Future Generat. Comput. Syst., 28: 58-65. DOI: 10.1016/j.future.2011.05.008

Venugopal, S. and R. Buyya, 2006. A set coveragebased mapping heuristic for scheduling distributed data-intensive applications on global grids. Proceedings of the 7th IEEE/ACM International Conference on Grid Computing, Sept. 28-29, IEEE Xplore Press, Barcelona, Spain, pp: 238-245. DOI: 10.1109/ICGRID.2006.311021

Verbelen, T., T. Stevens, F. De Turck and B. Dhoedt, 2013. Graph partitioning algorithms for optimizing software deployment in mobile cloud computing. Future Generat. Comput. Syst., 29: 451-459. DOI: 10.1016/j.future.2012.07.003

Vishwanath, K.V. and N. Nagappan, 2010. Characterizing cloud computing hardware reliability. Proceedings of the 1st ACM Symposium on Cloud Computing, Jun. 10-11, ACM, Indianapolis, Indiana, USA, pp: 193-204. DOI: $10.1145 / 1807128.1807161$

Waibel, P., C. Hochreiner and S. Schulte, 2016. Costefficient data redundancy in the cloud. Proceedings of the IEEE 9th International Conference on Service-Oriented Computing and Applications, Nov. 4-6, IEEE Xplore Press, Macau, China, pp: 1-9. DOI: $10.1109 /$ SOCA.2016.12

Wang, W., Y. Wang, X.Y. Li, W.Z. Song and O. Frieder, 2006. Efficient interference-aware TDMA link scheduling for static wireless networks. Proceedings of the 12th Annual International Conference on Mobile Computing and Networking, Sept. 23-29, ACM, Los Angeles, CA, USA, pp: 262-273. DOI: $10.1145 / 1161089.1161119$

Wang, X., T. Tiropanis and H.C. Davis, 2012. Evaluating graph traversal algorithms for distributed SPARQL query optimization. Proceedings of the Semantic Web: Joint International Semantic Technology Conference, Dec. 4-7, Springer, Hangzhou, China, pp: 210-225. DOI: 10.1007/978-3-642-29923-0_14 
Wang, Y. and X.Y. Li, 2006. Minimum power assignment in wireless ad hoc networks with spanner property, J. Combinat. Optimiz., 11: 99-112. DOI: $10.1023 / \mathrm{A}: 1012319418150$

Ward, J.S. and A. Barker, 2014. Observing the clouds: A survey and taxonomy of cloud monitoring. J. Cloud Comput.: Adv. Syst. Applic., 3: 24-24. DOI: $10.1186 / \mathrm{s} 13677-014-0024-2$

Warneke, D. and O. Kao, 2011. Exploiting dynamic resource allocation for efficient parallel data processing in the cloud. IEEE Trans. Parallel Distributed Syst., 22: 985-997. DOI: 10.1109/TPDS.2011.65

Wood, T., P. Shenoy, A. Venkataramani and M. Yousif, 2007. Black-box and gray-box strategies for virtual machine migration. Proceedings of the 4th USENIX Conference on Networked Systems Design and Implementation, Apr. 11-13, USENIX Association, Cambridge, MA, pp: 229-242.

Wu, C. and R. Buyya, 2015. Cloud Data Centers and Cost Modeling: A Complete Guide to Planning, Designing and Building a Cloud Data Center. 1st Edn., Morgan Kaufmann, Waltham, MA, ISBN-10: 012801413X, pp: 512.

$\mathrm{Wu}, \mathrm{J}$. and H. Li, 1999. On calculating connected dominating set for efficient routing in ad hoc wireless networks. Proceedings of the 3rd International Workshop on Discrete Algorithms and Methods for Mobile Computing and Communications, Aug. 2020, ACM, Seattle, Washington, USA, pp: 7-14. DOI: $10.1145 / 313239.313261$

Xiao, W. and B. Parhami, 2003. Some conclusions on cayley digraphs and their applications to interconnection networks. Proceedings of the 2nd International Conference on Grid and Cooperative Computing, Dec. 7-10, Springer, Shanghai, China, pp: 408-412. DOI: 10.1007/978-3-540-24680-0_67
Xie, G., R. Li, X. Xiao and Y. Chen, 2014. A highperformance DAG task scheduling algorithm for heterogeneous networked embedded systems. Proceedings of the IEEE 28th International Conference on Advanced Information Networking and Applications, May 13-16, IEEE Xplore Press, Victoria, BC, Canada, pp: 1011-1016. DOI: 10.1109/AINA.2014.123

Xie, W., M. Goyal, H. Hosseini, J. Martocci and Y. Bashir et al., 2010. Routing loops in DAG-based low power and lossy networks. Proceedings of the 24th IEEE International Conference on Advanced Information Networking and Applications, Apr. 20-23, IEEE Xplore Press, Perth, WA, Australia, pp: 888895. DOI: $10.1109 /$ AINA.2010.126

Zegzhda, D.P. and A.V. Nikolsky, 2014. Formal security model for virtual machine hypervisors in cloud computing systems. Nonlinear Phenomena Complex Syst., 17: 253-262.

Zhang, Q., L. Cheng and R. Boutaba, 2010. Cloud computing: State of-the-art and research challenges. J. Internet Services Applic., 1: 7-18. DOI: $10.1007 / \mathrm{s} 13174-010-0007-6$

Zheng, Z., T.C. Zhou, M.R. Lyu and I. King, 2012. Component ranking for fault-tolerant cloud applications. IEEE Trans. Services Comput., 5: 1097-1106. DOI: 10.1109/TSC.2011.42

Zhou, Y., O. Grygorash and T.F. Hain, 2011. Clustering with minimum spanning trees. Int. J. Artificial Intell. Tools, 20: 139-177. DOI: $10.1142 / \mathrm{S} 0218213011000061$

Zissis, D. and D. Lekkas, 2012. Addressing cloud computing security issues. Future Generat. Comput. Syst., 28: 583-592. DOI: 10.1016/j.future.2010.12.006 